\title{
STOCHASTIC APPROXIMATION TYPE ALGORITHM FOR THE MAXIMIZATION OF THE PROBABILITY FUNCTION
}

\author{
(Presented by N. Alumäe)
}

1. Introduction. In this paper the problem of maximizing the probability function

$$
v(x, t)=P[f(x, \xi)<t]
$$

is considered. Here $f(x, \xi)$ is a real-valued function defined on a Euclidean space $R^{r} \times R^{s}$; $\xi$, an $s$-dimensional random parameter; $x$, an $r$-dimensional control parameter; $t$, a fixed number and $P$ denotes probability. Optimization problems with probability function (1) arise in optimal programming, control and identification.

Consider the problem

$$
\max _{x \in R^{r}} P[f(x, \xi)<t]
$$

By the Heaviside function $\chi(y)$,

$$
\chi(y)= \begin{cases}0, & y \leqslant 0 \\ 1, & y>0\end{cases}
$$

we can present the problem (2) in the following manner:

$$
\max _{x \in R^{r}} \int_{\Xi} \chi(t-f(x, \xi)) d P(\xi) \text {. }
$$

Thus, the maximization of the probability function is formally a particular case of that of the mathematical expectation, and a solution of the problem (2) we can find by some stochastic approximation type method. However, the derivative in $x$ of the Heaviside function $\chi(t-f(x, \xi))$ is the Dirac $\delta$-function, which is not a function in usual sense. Under these circumstance we cannot use directly the well-known stochastic approximation methods. It seems quite likely to be a reason for the fact that there exist only very few methods for solving (2), e. g. in [ [1-4]. References in $\left[{ }^{2,3}\right]$ deal with a Kiefer-Wolfowitz type algorithm and rely on the Lipschitz condition of the $v_{x}^{\prime}(x, t)$. In [ [ ] the function $\chi(t-f(x, \xi))$ is estimated by a differentiable in $x$ Parzen kernel-type estimate, and in the $n$-th iteration $n$ realizations of the random parameter $\xi$ are used to calculate the estimate to the $v_{x}^{\prime}(x, t)$. In $\left[{ }^{5}\right]$ it is shown that the statistical estimation type methods have no advantages in asymptotic sence compared with methods of random search and at the same time require more calculating efforts. According to these considerations, in this paper we propose a random search algorithm based on realizations of Parzen kernel-type estimation.

2. The algorithm. Let $\xi_{n}$ be the $n$-th realization of the random vector $\xi$ and let the function $f(x, \xi)$ be differentiable in $x$ for almost all $\xi$. Let us determine the algorithm for the maximization of the probability function (1) in the following way: 


$$
x_{n+1}=x_{n}-\gamma_{n} h_{n}^{-1} f_{x}^{\prime}\left(x_{n}, \xi_{n}\right) K\left(\left(t-f\left(x_{n}, \xi_{n}\right)\right) h_{n}^{-1}\right) .
$$

To prove the convergence of this algorithm, the following assumptions are needed:

A 1. The kernel $K(y)$ is a continuous function with

$$
\begin{gathered}
\sup _{y}|K(y)| \leqslant K<\infty, \quad \int_{-\infty}^{\infty}|y K(y)| d y<\infty, \\
|y||K(y)| \rightarrow 0, \quad|y| \rightarrow 0 .
\end{gathered}
$$

A 2. The function $f(x, \xi)$ is differentiable in $x$ and $\xi$ and $\left\|f^{\prime}(x, \xi)\right\| \neq 0$ for almost all $\xi$ and all $x$.

A 3. Derivatives $v_{x}^{\prime}(x, t)$ and $v^{\prime \prime}{ }_{x t}(x, t)$ exist, are bounded and continuous in $x$ for $\|x\| \leqslant T$ and all $t \in R^{1}$.

A 4. The random vector $\xi$ has a density $p(\xi)$.

If the assumptions $\mathrm{A} 1-\mathrm{A} 4$ hold, then

$$
\begin{gathered}
E\left\{h_{n}^{-1} f_{x}^{\prime}\left(x_{n}, \xi_{n}\right) K\left(\left(t-f\left(x_{n}, \xi_{n}\right)\right) h_{n}^{-1}\right) \mid x_{0}, \ldots, x_{n}\right\}= \\
=h_{n}^{-1} \int_{R^{s}} f_{x}^{\prime}\left(x_{n}, \xi\right) K\left(\left(t-f\left(x_{n}, \xi\right)\right) h_{n}^{-1}\right) p(\xi) d \xi= \\
=h_{n}^{-1} \int_{-\infty}^{\infty} K\left((t-v) h_{n}^{-1}\right) \int_{S_{x, v}} f^{\prime} x_{x}\left(x_{n}, \xi\right)\left\|f^{\prime}\left(x_{n}, \xi\right)\right\|^{-1} p(\xi) d S_{x, v} d v= \\
=h_{n}^{-1} \int_{-\infty}^{\infty} K\left((t-v) h_{n}^{-1}\right) v_{x}^{\prime}\left(x_{n}, v\right) d v= \\
=\int_{-\infty}^{\infty} K(z) v_{x}^{\prime}\left(x_{n}, t-h_{n} z\right) d z,
\end{gathered}
$$

where $S_{x, v}=\{\xi: f(x, \xi)=v\}$ and (due to $\left[{ }^{1}\right]$ )

$$
v_{x}^{\prime}(x, t)=\int_{S_{x, v}} f_{x}^{\prime}(x, \xi)\left\|f_{\xi}^{\prime}(x, \xi)\right\|^{-1} p(\xi) d S_{x, v} .
$$

By A 3

$$
v_{x}^{\prime}\left(x_{n}, t-h_{n} z\right)=v_{x}^{\prime}\left(x_{n}, t\right)-h_{n} z v_{x t}^{\prime \prime}\left(x_{n}, t-\theta_{n} h_{n} z\right)
$$

and now

$$
\begin{gathered}
E\left\{h_{n}^{-1} f_{x}^{\prime}\left(x_{n}, \xi_{n}\right) K\left(\left(t-f\left(x_{n}, \xi_{n}\right)\right) h_{n}^{-1}\right) \mid x_{0}, \ldots, x_{n}\right\}= \\
=v_{x}^{\prime}\left(x_{n}, t\right)-h_{n} \int_{-\infty}^{\infty} z K(z) v_{x t}^{\prime \prime}\left(x_{n}, t-\theta_{n} h_{n} z\right) d z .
\end{gathered}
$$

Under the conditions A 1 and A 3, the integral in the last expression is bounded for $\|x\| \leqslant T$, and if $h_{n}$ tends to zero then $h_{n}^{-1} f^{\prime}{ }_{x}\left(x_{n}, \xi_{n}\right) K X$ $\times\left(\left(t-f\left(x_{n}, \xi_{n}\right)\right) h_{n}^{-1}\right)$ is a stochastic quasigradient of $v(x, t)$ (see $\left.\left[{ }^{6}\right]\right)$.

Let $X^{*}$ be the set of stationary points, i.e.

$$
X^{*}=\left\{x^{*}: v_{x}^{\prime}\left(x^{*}, t\right)=0\right\} .
$$

According to $\left[{ }^{7}\right]$, we can say that the method is convergent if all the limit points of the sequence $\left(x_{n}\right)$ belong to the set $X^{*}$ with probability 1 (w. p. 1).

3. Theorem of convergence. In order to formulate and prove 
the theorem of convergence, the following additional assumptions are needed:

A 5. $E\left\|f^{\prime}{ }_{x}(x, \xi)\right\|^{2}$ is bounded for $\|x\| \leqslant T$.

A 6. $\gamma_{n} \rightarrow 0, \quad h_{n} \rightarrow 0, \quad \gamma_{n} h_{n}^{-1} \rightarrow 0$,

$$
\begin{array}{ll}
\sum_{n=1}^{\infty} \gamma_{n}=\infty, & \sum_{n=1}^{\infty} \gamma_{n}^{2}<\infty, \\
\sum_{n=1}^{\infty} \gamma_{n} h_{n}<\infty, & \sum_{n=1}^{\infty} \gamma_{n}^{2} h_{n}^{-2}<\infty .
\end{array}
$$

A7. The function $v(x, t)$ takes no more than a countable number of values on the set $X^{*}$.

Theorem. Assume that the conditions $\mathrm{A} 1-\mathrm{A} 7$ are satisfied and sup $\left\|x_{n}\right\|<\infty$ w. p. 1 . Then all the limit points of the sequence $\left(x_{n}\right)$ belong to the set $X^{*}$ w.p. 1 .

The proof relies on the following

Le m m a $\left.{ }^{7}\right]$. Let a random sequence $\left(x_{n}(\omega)\right)$ and the solution set $X^{*}$ satisfy the following conditions

1) there exists such a closed and bounded set $S$ that $\left(x_{n}(\omega)\right) \in S$ w. p. 1;

2) for any convergent subsequence $\left(x_{n_{k}}(\omega)\right)$

a) if $\lim _{h \rightarrow \infty} x_{n_{k}}(\omega) \in X^{*}$ w. p. 1, then

$$
\lim _{k \rightarrow \infty}\left\|x_{n_{k+1}}(\omega)-x_{n_{k}}(\omega)\right\|=0 \quad \omega . p .1,
$$

b) if $\lim _{k \rightarrow \infty} x_{n_{k}}(\omega)=x^{\prime}(\omega) \notin X^{*}$ for $\omega \in B, P(B)>0$, then there exists such a real-valued random variable $\varepsilon_{0}(\omega)$ that for all $k$ and $\varepsilon(\omega)$, $0<\varepsilon(\omega) \leqslant \varepsilon_{0}(\omega)$, the variable $\tau_{k}(\omega)$ is bounded for $\omega \in B$, where

$$
\tau_{k}(\omega)=\min _{n>n_{k}(\omega)}\left\{n:\left\|x_{n}-x_{n_{k}}\right\|>\varepsilon\right\} ;
$$

3) there exists such a continuous function $W(x)$ that for all $\omega \in B$

$$
\varlimsup_{k \rightarrow \infty} W\left(x_{\tau_{k}}(\omega)\right)<\lim _{k \rightarrow \infty} W\left(x_{n_{k}}(\omega)\right) ;
$$

4) the function $W(x)$ takes no more than a countable number of values on the set $X^{*}$;

then the limit of every convergent subsequence of the sequence $\left(x_{n}\right)$ belongs to the set $X^{*} w . p .1$.

Proof of the theorem. Test the conditions of the Lemma. Suppose that there is some real $T$ for which $\left\|x_{n}\right\| \leqslant T$ w. p. 1 for all $n$. The condition 2a) is fulfilled by A $1, \mathrm{~A} 6$ and A 7 . If $2 \mathrm{~b}$ ) is not fulfilled, then for $s \geqslant n_{k}, \quad\left\|x_{s}-x_{n_{k}}\right\|<\varepsilon, \quad\left\|x_{n_{k}}-x^{\prime}\right\|<\varepsilon \quad$ and $\quad\left\|x_{s}-x^{\prime}\right\| \leqslant\left\|x_{s}-x_{n_{k}}\right\|+$ $+\left\|x_{n_{k}}-x^{\prime}\right\|<2 \varepsilon$. Taking $W(x)=v(x, t)$ we get

$$
v\left(x_{s}, t\right)-v\left(x_{n_{k}}, t\right)=\left(v_{x}^{\prime}\left(x_{n_{k}}, t\right), x_{s}-x_{n_{k}}\right)+o(\varepsilon)
$$

and

Then

$$
x_{s}-x_{n_{k}}=-\sum_{n=n_{k}}^{s-1} \gamma_{n} h_{n}^{-1} f^{\prime}\left(x_{n}, \xi_{n}\right) K\left(\left(t-f\left(x_{n}, \xi_{n}\right)\right) h_{n}^{-1}\right) .
$$

$$
v\left(x_{s}, t\right)-v\left(x_{n_{k}}, t\right)=-\left(v_{x}^{\prime}\left(x_{n_{k}}, t\right),\right.
$$


$\left.\sum_{n=n_{k}}^{s-1} \gamma_{n} h_{n}^{-1} f_{x}^{\prime}\left(x_{n}, \xi_{n}\right) K\left(\left(t-f\left(x_{n}, \xi_{n}\right)\right) h_{n}^{-1}\right)\right)+o(\varepsilon)=-\left(v_{x}^{\prime}\left(x_{n_{k}}, t\right)\right.$

$$
\left.\sum_{n=n_{k}}^{s-1} \gamma_{n} v_{x}^{\prime}\left(x_{n}, t\right)\right)+\left(v_{x}^{\prime}\left(x_{n_{k}}, t\right)\right.
$$

$$
\begin{gathered}
\sum_{n=n_{k}}^{s-1} \gamma_{n}\left[v_{x}^{\prime}\left(x_{n}, t\right)-h_{n} \int_{-\infty}^{\infty} z K(z) v_{x t}^{\prime \prime}\left(x_{n}, t-\theta_{n} h_{n} z\right) d z-\right. \\
\left.\left.-h_{n}^{-1} f_{x}^{\prime}\left(x_{n}, \xi_{n}\right) K\left(\left(t-f\left(x_{n}, \xi_{n}\right)\right) h_{n}^{-1}\right)\right]\right)+\left(v_{x}^{\prime}\left(x_{n_{k}}, t\right),\right. \\
\left.\sum_{n=n_{k}}^{s-1} \gamma_{n} h_{n} \int_{-\infty}^{\infty} z K(z) v_{x t}^{\prime \prime}\left(x_{n}, t-\theta_{n} h_{n} z\right) d z\right)+o(\varepsilon) .
\end{gathered}
$$

By (4)

$$
\begin{gathered}
E\left\{v_{x}^{\prime}\left(x_{n}, t\right)-h_{n} \int_{-\infty}^{\infty} z K(z) v_{x t}^{\prime \prime}\left(x_{n}, t-\theta_{n} h_{n} z\right) d z-\right. \\
\left.-h_{n}^{-1} f_{x}^{\prime}\left(x_{n}, \xi_{n}\right) K\left(\left(t-f\left(x_{n}, \xi_{n}\right)\right) h_{n}^{-1}\right) \mid x_{0}, \cdots, x_{n}\right\}=0 .
\end{gathered}
$$

Now consider the sum

$$
\sum_{n=n_{k}}^{\infty} E \| \gamma_{n} v^{\prime}{ }_{x}\left(x_{n}, t\right)-\gamma_{n} h_{n} \int_{-\infty}^{\infty} z K(z) v_{x t}^{\prime \prime}\left(x_{n}, t-\theta_{n} h_{n} z\right) d z-
$$

$-\gamma_{n} h_{n}^{-1} f_{x}^{\prime}\left(x_{n}, \xi_{n}\right) K\left(\left(t-f\left(x_{n}, \xi_{n}\right)\right) h_{n}^{-1}\right) \|^{2}=$

$=\sum_{n=n_{k}}^{\infty} \gamma_{n}^{2}\left\|v_{x}^{\prime}\left(x_{n}, t\right)\right\|^{2}+\sum_{n=n_{k}}^{\infty} \gamma_{n}^{2} h_{n}^{2}\left\|\int_{-\infty}^{\infty} z K(z) v_{x t}^{\prime \prime}\left(x_{n}, t-\theta_{n} h_{n} z\right) d z\right\|^{2}+$

$+\sum_{n=n_{k}}^{\infty} \gamma_{n}^{2} h_{n}^{-2} E\left\|f^{\prime}{ }_{x}\left(x_{n}, \xi_{n}\right) K\left(\left(t-f\left(x_{n}, \xi_{n}\right)\right) h_{n}^{-1}\right)\right\|^{2}-$

$-2 \sum_{n=n_{k}}^{\infty} \gamma_{n}^{2} h_{n}\left(v_{x}^{\prime}\left(x_{n}, t\right), \int_{-\infty}^{\infty} z K(z) v_{x t}^{\prime \prime}\left(x_{n}, t-\theta_{n} h_{n} z\right) d z\right)-$

$-2 \sum_{n=n_{k}}^{\infty} \gamma_{n}^{2} h_{n}^{-1}\left(v_{x}^{\prime}\left(x_{n}, t\right), f_{x}^{\prime}\left(x_{n}, \xi_{n}\right)-h_{n} \int_{-\infty}^{\infty} z K(z) v_{x t}^{\prime \prime}\left(x_{n}, t-\theta_{n} h_{n} z\right) d z\right)+$

$+2 \sum_{n=n_{k}}^{\infty} \gamma_{n}^{2}\left(\int_{-\infty}^{\infty} z K(z) v_{x t}^{\prime \prime}\left(x_{n}, t-\theta_{n} h_{n} z\right) d z, v_{x}^{\prime}\left(x_{n}, t\right)-\right.$

$\left.-h_{n} \int_{-\infty}^{\infty} z K(z) v_{x t}^{\prime \prime}\left(x_{n}, t-\theta_{n} h_{n} z\right) d z\right)<\infty$

due to

$$
E\left\|f_{x}^{\prime}\left(x_{n}, \xi_{n}\right) K\left(\left(t-f\left(x_{n}, \xi_{n}\right)\right) h_{n}^{-1}\right)\right\|^{2} \leqslant K^{2} E\left\|f^{\prime}{ }_{x}\left(x_{n}, \xi_{n}\right)\right\|^{2}
$$

and assumptions A 6 , sup $|K(y)| \leqslant K$.

Hence,

$$
\sum_{n=n_{k}}^{\infty} \gamma_{n}\left[v_{x}^{\prime}\left(x_{n}, t\right)+h_{n} \int_{-\infty}^{\infty} z K(z) v_{x t}^{\prime \prime}\left(x_{n}^{\prime}, t-\theta_{n} h_{n} z\right) d z !\right.
$$




$$
\left.-h_{n}^{-1} f^{\prime}{ }_{x}\left(x_{n}, \xi_{n}\right) K\left(\left(t-f\left(x_{n}, \xi_{n}\right)\right) h_{n}^{-1}\right)\right]<\infty \text { w. p. } 1 .
$$

Similarly $\sum_{n=n_{k}}^{\infty} \gamma_{n} h_{n} \int_{-\infty}^{\infty} z K(z) v_{x t}^{\prime \prime}\left(x_{n}, t-\theta_{n} h_{n} z\right) d z<\infty$. Since $x^{\prime} \notin X^{*}$, then $\left\|v_{x}^{\prime}\left(x^{\prime}, t\right)\right\|^{2}>\delta>0$ and as $v_{x}^{\prime}(x, t)$ is continuous in $x$ we can find a number $k$ such that $\left(v_{x}^{\prime}\left(x_{n_{k}}, t\right), v_{x}^{\prime}\left(x_{n}, t\right)\right)>\delta / 2$ for every $n \geqslant n_{k}$.

Hence

$$
\begin{gathered}
v\left(x_{s}, t\right)-v\left(x_{n_{k}}, t\right) \leqslant-\delta / 2 \sum_{n=n_{k}}^{s-1} \gamma_{n}+\left(v_{x}^{\prime}\left(x_{n_{k}}, t\right), \sum_{n=n_{k}}^{s-1} \gamma_{n}\left[v_{x}^{\prime}\left(x_{n}, t\right)-\right.\right. \\
\left.-h_{n} \int_{-\infty}^{\infty} z K(z) v_{x t}^{\prime \prime}\left(x_{n}, t-\theta_{n} h_{n} z\right) d z-h_{n}^{-1} f_{x}^{\prime}\left(x_{n}, \xi=K\left(\left(t-f\left(x_{n}, \xi_{n}\right)\right) / h_{n}\right)\right]\right)+ \\
\quad+\left(v_{x}^{\prime}\left(x_{n_{k}}, t\right), \sum_{n=n_{k}}^{s-1} \gamma_{n} h_{n} \int_{-\infty}^{\infty} z K(z) v_{x t}^{\prime \prime}\left(x_{n}, t-\theta_{n} h_{n} z\right) d z\right)+o(\delta)
\end{gathered}
$$

Going over to the limit by $s$, we reach a contradiction with the boundedness of $v\left(x_{s}, t\right)$.

So $2 \mathrm{~b}$ ) is proved.

Prove 3). Let $\tau_{k}=\min _{n>n_{k}}\left\{n:\left\|x_{n}-x_{n_{k}}\right\|>\varepsilon\right\}$. By the definition of $\tau_{k}$,

$$
\left\|x_{\tau_{\kappa}}-x_{n_{\kappa}}\right\|>\varepsilon
$$

and $\left\|x_{\tau_{k}-1}-x_{n_{k}}\right\| \leqslant \varepsilon$. Since $\gamma_{n} h_{n}^{-1} \rightarrow 0$ and $f_{x}^{\prime}\left(x_{n}, \xi_{n}\right) K\left(\left(t-f\left(x_{n}, \xi_{n}\right)\right) h_{n}^{-1}\right)$ is bounded w. p. 1 , so we can find such an index $k$ that

$$
\left\|x_{\tau_{k}}-x_{n_{k}}\right\| \leqslant\left\|x_{\tau_{k}}-x_{\tau_{k}-1}\right\|+\left\|x_{\tau_{k}-1}-x_{n_{k}}\right\|<2 \varepsilon .
$$

Therefore, the inequality (6) holds for $s=\tau_{k}$ as well, i. e.

$$
\begin{aligned}
& v\left(x_{\tau_{k}}, t\right)-v\left(x_{n_{k}}, t\right) \leqslant-\delta / 2 \sum_{n=n_{k}}^{\tau_{k}-1} \gamma_{n}+\left(v_{x}^{\prime}\left(x_{n_{k}}, t\right), \sum_{n=n_{k}}^{\tau_{k}-1} \gamma_{n}\left[v_{x}^{\prime}\left(x_{n}, t\right)-\right.\right. \\
& -h_{n} \int_{-\infty}^{\infty} z K(z) v_{x t}^{\prime \prime}\left(x_{n}, t-\theta_{n} h_{n} z\right) d z- \\
& \left.\left.-h_{n}^{-1} f_{x}^{\prime}\left(x_{n}, \xi_{n}\right) K\left(\left(t-f\left(x_{n}, \xi n\right)\right) h_{n}^{-1}\right)\right]\right)+ \\
& +\left(v_{x}^{\prime}\left(x_{n_{k}}, t\right), \sum_{n=n_{k}}^{\tau_{k}-1} \gamma_{n} h_{n} \int_{-\infty}^{\infty} z K(z) v_{x t}^{\prime \prime}\left(x_{n}, t-\theta_{n} h_{n} z\right) d z\right)+o(\varepsilon) .
\end{aligned}
$$

From (7) we obtain

$$
\begin{gathered}
\mathrm{\varepsilon}<\left\|x_{\tau_{k}}-x_{n_{k}}\right\| \leqslant \sum_{n=n_{k}}^{\tau_{k}-1}\left\|x_{n+1}-x_{n}\right\|= \\
=\sum_{n=n_{k}}^{\tau_{k}-1} \gamma_{n} h_{n}^{-1}\left\|f^{\prime}{ }_{x}\left(x_{n}, \xi_{n}\right) K\left(\left(t-f\left(x_{n}, \xi_{n}\right)\right) h_{n}^{-1}\right)\right\|
\end{gathered}
$$

and according to (5)

$$
\sum_{n=n_{k}}^{\infty} \gamma_{n} h_{n}^{-1}\left\|f^{\prime} x\left(x_{n}, \xi_{n}\right) K\left(\left(t-f\left(x_{n}, \xi_{n}\right)\right) h_{n}^{-1}\right)\right\|<\infty \quad \text { w. p. } 1 .
$$


So $h_{n}^{-1}\left\|f^{\prime}{ }_{x}\left(x_{n}, \dot{\xi}_{n}\right) \ddot{K}\left(\left(t-f\left(x_{n}, \xi_{n}\right)\right) h_{n}^{-1}\right)\right\|$ is bounded with some $M$ w.p. 1 . Therefore $\sum_{n=n_{k}}^{\tau_{k}-1} \gamma_{n} \geqslant \varepsilon / M$ and from (8) we get

$$
\begin{aligned}
& v\left(x_{\tau_{k}}, t\right)-v\left(x_{n_{k}}, t\right) \leqslant-\delta \varepsilon / 2 M+\left\|v_{x}^{\prime}\left(x_{n_{k}}, t\right)\right\| \| \sum_{n=n_{k}}^{\tau_{k}-1} \gamma_{n}\left[v_{x}^{\prime}\left(x_{n}, t\right)-\right. \\
& -h_{n} \int_{-\infty}^{\infty} z K(z) v_{x t}^{\prime \prime}\left(x_{n}, t-\theta_{n} h_{n} z\right) d z- \\
& \left.-h_{n}^{-1} f_{x}^{\prime}\left(x_{n}, \xi_{n}\right) K\left(\left(t-f\left(x_{n}, \xi_{n}\right)\right) h_{n}^{-1}\right)\right] \|+ \\
& +\left\|v_{x}^{\prime}\left(x_{n_{k}}, t\right)\right\|\left\|\sum_{n=n_{k}}^{\tau_{k}-1} \gamma_{n} h_{n} \int_{-\infty}^{\infty} z K(z) v_{x t}^{\prime \prime}\left(x_{n}, t-\theta_{n} h_{n} z\right) d z\right\|+o(\varepsilon) .
\end{aligned}
$$

From (5); we obtain

$$
\begin{gathered}
\lim _{\substack{h \rightarrow \infty \\
m \rightarrow \infty}} \sum_{n=k}^{m} \gamma_{n}\left[v_{x}^{\prime}\left(x_{n}, t\right)-h_{n} \int_{-\infty}^{\infty} z K(z) v_{x t}^{\prime \prime}\left(x_{n}, t-\theta_{n} h_{n} z\right) d z-\right. \\
\left.-h_{n}^{-1} f_{x}^{\prime}\left(x_{n}, \xi_{n}\right) K\left(\left(t-f\left(x_{n}, \xi_{n}\right)\right) h_{n}^{-1}\right)\right] \|=0
\end{gathered}
$$

and

$$
\lim _{\substack{k \rightarrow \infty \\ m \rightarrow \infty}}\left\|\sum_{n=k}^{m} \gamma_{n} h_{n} \int_{-\infty}^{\infty} z K(z) v_{x t}^{\prime \prime}\left(x_{n}, t-\theta_{n} h_{n} z\right) d z\right\|=0 .
$$

The limits (9) and (10) are also true for $m=\tau_{k}-1$ and $k=n_{k}$, and hence,

$$
\varlimsup_{k \rightarrow \infty} v\left(x_{\tau_{k}}, t\right) \leqslant \lim _{k \rightarrow \infty} v\left(x_{n_{k}}, t\right)-\delta \varepsilon / 2 M .
$$

So 3 ) is proved.

\begin{tabular}{|c|c|c|c|c|}
\hline & $\gamma_{n}=100 / n^{4 / 5}$ & $h_{n}=1 / n^{1 / 4}$ & $\gamma_{n}=100 / n^{6 / 7}$ & $h_{n}=1 / n^{1 / 5}$ \\
\hline$n$ & $x_{1, n}$ & $x_{2 n}$ & $x_{1 n}$ & $x_{2 n}$ \\
\hline $\begin{array}{r}25 \\
50 \\
75 \\
100 \\
125 \\
150 \\
175 \\
200 \\
225 \\
250\end{array}$ & $\begin{array}{l}4,3700 \\
3,8564 \\
3,3437 \\
2,6680 \\
1,7800 \\
6,4830 \cdot 10^{-4} \\
-2,5653 \cdot 10^{-5} \\
-2,2580 \cdot 10^{-9} \\
\quad- \\
-\end{array}$ & $\begin{array}{l}3,4960 \\
3,0850 \\
2,6750 \\
2,1344 \\
1,4240 \\
5,1863 \cdot 10^{-4} \\
-2,0523 \cdot 10^{-5} \\
-1,8064 \cdot 10^{-9} \\
\quad-\end{array}$ & $\begin{array}{l}4,3535 \\
3,9835 \\
3,6864 \\
3,4302 \\
3,1760 \\
2,8330 \\
2,3060 \\
1,5992 \\
-1,0348 \cdot 10^{-2} \\
-1,0380 \cdot 10^{-6}\end{array}$ & $\begin{array}{l}3,4828 \\
3,1868 \\
2,9492 \\
2,7442 \\
2,5408 \\
2,2663 \\
1,8447 \\
1,2794 \\
-8,2787 \cdot 10^{-3} \\
-8,3040 \cdot 10^{-7}\end{array}$ \\
\hline
\end{tabular}

The proof with the assumption sup $\left\|x_{n}\right\|<\infty \quad$ w. p. 1 instead of the boundedness of $T$ proceeds similarly. We repeat about the proof, but stop the iteration $\left(x_{n}\right)$ at the first moment when $\left\|x_{n}\right\|>T$. Then we conclude that $x_{n} \rightarrow x^{*}$ with a probability $\geqslant P\left[\sup \left\|x_{n}\right\| \leqslant T\right]$. Since $T$ is arbitrary, the theorem holds as stated. Q.E.D.

4. Numerical example. Consider the problem

Table $\mathbf{i}$ 


\begin{tabular}{|c|c|c|c|c|}
\hline \multirow[b]{2}{*}{$n$} & \multicolumn{2}{|c|}{${ }^{a} \gamma_{n}=100 / n^{3 / 4}, \quad h_{n}=1 / n^{1 / 2}$} & \multicolumn{2}{|c|}{${ }^{b} \gamma_{n}=10 / n^{3 / 4}, \quad h_{n}=1 / n^{1 / 3}$} \\
\hline & $x_{1 n}$ & $x_{2 n}$ & $x_{1 n}$ & $x_{2 n}$ \\
\hline 25 & 2,6221 & 2,0977 & 4,9185 & 3,9348 \\
\hline 50 & 3,1425 & 2,5140 & 4,8703 & 3,9035 \\
\hline 75 & 1,9844 & 1,5875 & 4,8585 & 3,8868 \\
\hline 100 & $3,8810 \cdot 10^{-2}$ & $3,1047 \cdot 10^{-2}$ & 4,8370 & 3,8696 \\
\hline 125 & $-3,7310 \cdot 10^{-4}$ & $-2,9847 \cdot 10^{-4}$ & 4,8181 & 3,8545 \\
\hline 150 & $-0,2640$ & $-0,2112$ & 4,8000 & 3,8400 \\
\hline 175 & $4,8093 \cdot 10^{-2}$ & $3,8474 \cdot 10^{-2}$ & 4,7818 & 3,8254 \\
\hline 200 & 3,1122 & 2,4898 & 4,7690 & 3,8095 \\
\hline$\overline{725}$ & $-2,12 \overline{8} \cdot 10^{-2}$ & $-1.703 \overline{1} \cdot 10^{-2}$ & $4 \overline{5687}$ & $3 . \overline{6550}$ \\
\hline 750 & 2,5470 & 2,3376 & 4,5580 & 3,6464 \\
\hline
\end{tabular}

where $\xi_{1}$, and $\xi_{2}$ are uniformly in $[0,1]$ distributed random parameters,

$$
K(y)=(2 \pi)^{-1 / 2} \exp \left(-y^{2} / 2\right), \quad x_{0}=(5 ; 4) .
$$

The solution of the problem is $(0,0)$. The results of computing are presented in Tables 1 and 2. In Tabl. 2a the assumption $\sum_{n=1}^{\infty} \gamma_{n}^{2} / h_{n}^{-2}<\infty$ is not fulfilled. Since the function $v(x, t)$ is quite aslant, we need large values for $\gamma_{n}$ at the beginning. Tabl. 2b shows that for smaller values of $\gamma_{n}$ the convergence of $\left(x_{n}\right)$ is comparatively slow.

\section{REFERENCES}

1. Райк Э. Изв. АН ЭССР, Физ. Матем., 24, № 1, 3-9 (1975).

2. Юби Э. Тр. Таллин. политехн. ин-та, № 411, 57-76 (1976).

3. Юби Э. Тр. Таллин. политехн. нн-та, № 482, 121-130 (1980).

4. Лenn P. Изв. АН ЭССР, Физ. Матем., 28, № 4, 303-309 (1979).

5. Поляк Б. Т., Цыпкин Я. З. Автоматика и телемеханика, № 8, 74-84 (1980).

6. Ермольев Ю. М. Методы стохастического программирования, М., «Наука», 1976.

7. Нурминский $E$. A. Численные методы решения детерминированных и стохастических минимаксных задач. Киев, «Наукова думка», 1979.

Academy of Sciences of the Estonian SSR, Institute of Cybernetics

Received June 18,1982

\section{R. LEPP}

\section{TÕENÄOSUSFUNKTSIOONI MAKSIMEERIMINE STOHHASTILISE APROKSIMATSIOONI TUOPI MEETODI ABIL}

Tōenäosusfunktsiooni $v(x, t)=P[f(x, \xi)<t]$ maksimeerimiseks on kasutatud stohhastilise aproksimatsiooni tüüpi meetodit, kus tõenäosusfunktsiooni $v(x, t)$ gradient asendatakse igal iteratsioonisammul Parzeni tuuma tüüpi hinnangu realisatsiooniga. On näidatud, et viimane on funktsiooni $v_{x}^{\prime}(x, t)$ kvaasigradient, ja tõestatud meetodi koonduṿust.

Р. ЛЕПП

\section{МЕТОД ТИПА СТОХАСТИЧЕСКОЙ АППРОКСИМАЦИИ ДЛЯ МАКСИМИЗАЦИИ ФУНКЦИИ ВЕРОЯТНОСТИ}

Для максимизации функции вероятности $v(x, t)=P[f(x, \xi)<t]$ предлагается метод типа стохастической аппроксимации. На каждом итерационном шаге $v^{\prime}{ }_{x}(x, t)$ заменяется одной реализацией ее парзеновской оценки. Показывается, что метод является квазиградиентным, и доказывается его сходимость. 